\title{
A Comparison of Dissolution Testing on Lipid Soft Gelatin Capsules Using USP Apparatus 2 and Apparatus 4
}

\author{
Jack Hu' ${ }^{1,2}$, Ali Kyad ${ }^{1}$, Vivian Ku ${ }^{1}$, Peter Zhou ${ }^{1}$, Nina Cauchon ${ }^{1}$
}

email:jhu@amgen.com

\begin{abstract}
The USP Apparatus 2 and Apparatus 4 were compared for dissolution of soft gelatin capsule formulations of a poorly watersoluble amine drug. Data is presented for the solubility of the drug in acidic media - with and without added surfactant. A $0.01 \mathrm{~N} \mathrm{HCl} / 0.25 \%$ Polysorbate 80 medium was selected for further development.

When the soft gelatin capsule ruptures during dissolution, the oil phase is released into the aqueous medium. Both dissolution methods were adapted to handle the problems caused by the oil phase passing into the filters and sample collection system.

Using this acidic medium with added surfactant, both apparatuses gave similar dissolution profiles. Apparatus 2 tended to give a faster rate of dissolution, but Apparatus 4 was better able to distinguish between different formulations.
\end{abstract}

\section{Introduction}

oft gelatin capsule (SGC) formulations are becoming

more popular in recent years $(1,2)$. These formulations

can mask odors and unpleasant tastes, and are easy to

swallow. They are suitable for encapsulation of lipid solu-

tions, suspensions, or paste-like formulations, making them a useful option when formulating poorly water-soluble drugs.

Due to the unique properties of SGC formulations, there exist many challenges to development of a good dissolution method. Generally, the drug is dissolved or suspended in oil before encapsulation. During dissolution, the oil may form a layer above the aqueous medium. In addition, oil droplets may become suspended in the medium. The existence of the lipid phase may hinder the release of the drug, and the oil layer or oil droplets may cause sampling problems.

Furthermore, poor aqueous solubility of the drug may cause slow or incomplete dissolution.

Initially, solubility studies on the drug substance were carried out resulting in a selection of suitable dissolution media. Dissolution experiments were tried using USP Apparatus 1,2 , and 4 . Since the dissolution rate was too slow using USP Apparatus 1, only Apparatus 2 and 4 were selected for further method development.

During the method development, the sampling problems when using USP Apparatus 2 had to be resolved and a suitable flow cell chosen for Apparatus 4. Once these changes were made, dissolution profiles were obtained using both apparatuses for the two SGC formulations. The results were analyzed and compared.

\section{Background information}

The drug substance in this article is the free base of a secondary amine. The pKa is about 8.4. The solubility in water is low, but it is freely soluble in soybean oil.

Figure 1 shows the solubility of the hydrochloride salt of the free base in $\mathrm{HCl}$. A concentration of $0.01 \mathrm{~N} \mathrm{HCl}$ was selected for further development. The effect of the surfactant Polysorbate 80 was also explored. Figure 2 shows the

Table 1. Dissolution Conditions Used with Apparatus 2 and Apparatus 4.

\begin{tabular}{|l|c|c|}
\hline \multicolumn{1}{|c|}{$\begin{array}{c}\text { Equipment } \\
\text { and parameters }\end{array}$} & $\begin{array}{c}\text { Apparatus 2 } \\
\text { (paddles) }\end{array}$ & $\begin{array}{c}\text { Apparatus 4 } \\
\text { (flow-through cells) }\end{array}$ \\
\hline Apparatus & $\begin{array}{c}\text { DISTEK 2100B Dissolution System equipped } \\
\text { with HP 8453 online UV spectrophotometer }\end{array}$ & $\begin{array}{c}\text { Sotax CE7 Dissolution System equipped with } \\
\text { Softgel Capsule Flow-through Cells }\end{array}$ \\
\hline Filters & Filter tip & Glass Microfiber Filter \\
\hline Temperature & $37^{\circ} \mathrm{C}$ & $37^{\circ} \mathrm{C}$ \\
\hline Paddle rotation & $50 \mathrm{RPM}$ & $16 \mathrm{~mL} / \mathrm{minute}$ \\
\hline Flow Rate & - & Offline UV at $273 \mathrm{~nm}$ \\
\hline Detection & UV at 273 nm & \\
\hline $\begin{array}{l}\mathrm{RPM}=\text { revolutions per minute } \\
\mathrm{HCl}=\text { hydrochloric acid }\end{array}$ & & \\
\hline
\end{tabular}

'Pharmaceutics Department, Amgen Inc., One Amgen Center Drive,

${ }^{2}$ Corresponding author.

Thousand Oaks, CA 91320 USA 


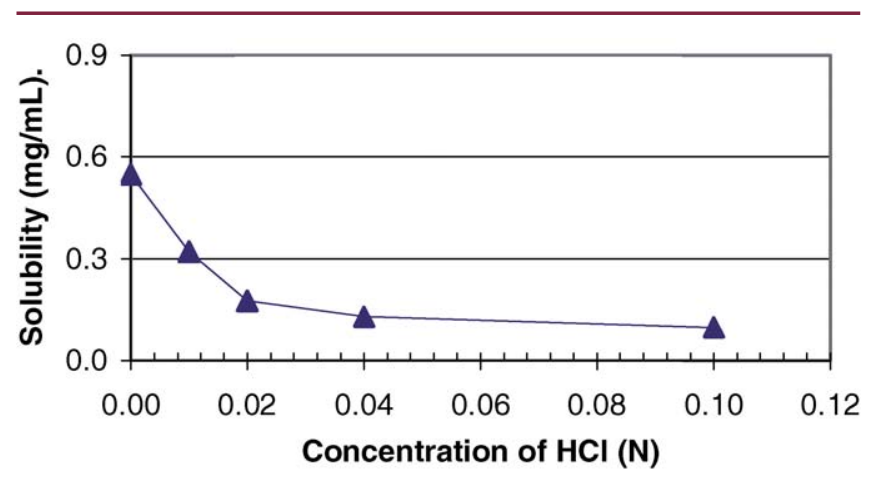

Figure 1. Solubility of Drug Substance in Dilute $\mathrm{HCl}$ Solution

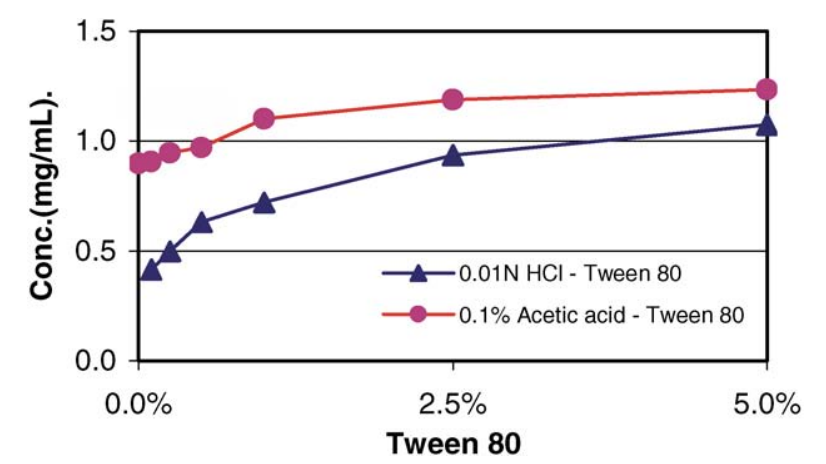

Figure 2. Solubility of Drug Substance in Hydrochloric and Acetic Acids with Polysorbate 80

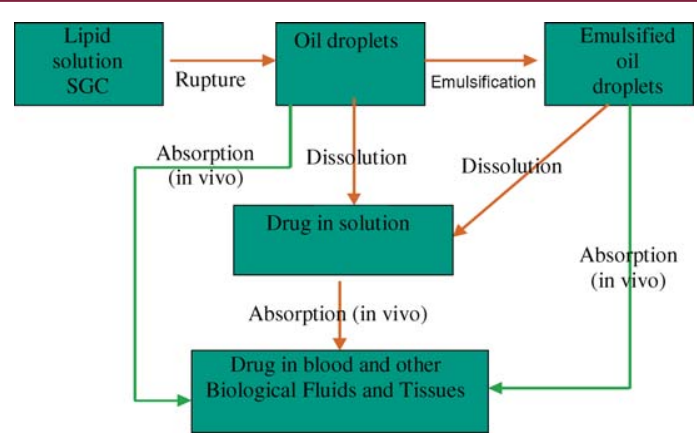

Figure 3. Schematic Diagram of Lipid Solution SGC Dissolution Process

solubility in $0.01 \mathrm{~N} \mathrm{HCl}$ and $0.1 \%$ acetic acid in the presence of Polysorbate 80 (Tween 80 ).

Two SGC formulations were used for this study. In each case, the drug load was about $30 \%$, and the amounts of the additives and the oil phase were equivalent for both formulations. Formulation A contained soybean oil and Formulation B contained glyceryl caprylate.

The conditions used with the two apparatuses are compared in Table 1.

Figure 3 shows a schematic diagram of the dissolution process for a lipid solution filled soft gelatin capsule. It is similar to the schematic illustration of the dissolution process for solids (3).

Figure 4 is a schematic illustration of the extraction process of the drug from the oil phase into the dissolution medium.

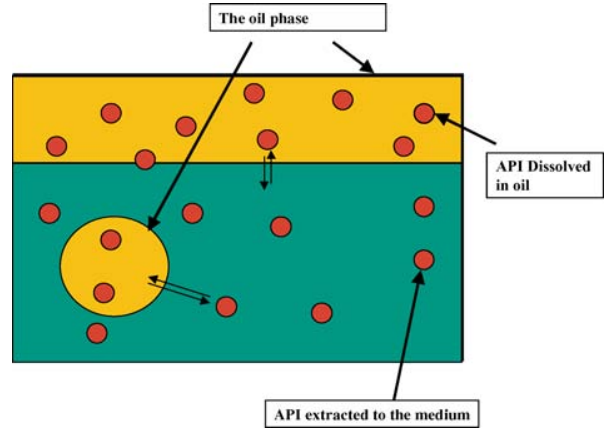

Figure 4. Schematic Illustration of Extraction Process

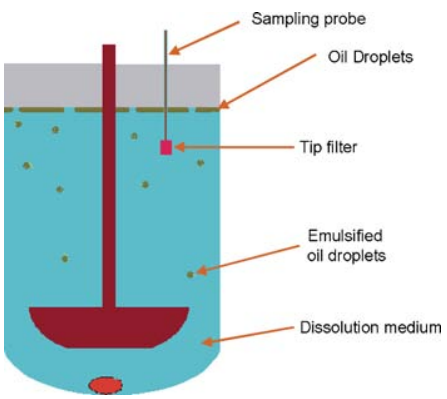

Figure 5. Schematic Diagram of a SGC in USP Apparatus 2 Dissolution Vessel

\section{Dissolution using USP Apparatus 2}

Initial dissolution method development was performed using USP Apparatus 2. During these exploratory studies, it was found that after the SGC ruptured, the contents floated to the top of the dissolution medium, forming oil droplets. Conventionally, when using USP Apparatus 2, the sampling probe is immersed in the dissolution medium during the sampling, and lifted up above the medium after each sampling time point. During this sampling process, the filter tip on the probe passed through the oil phase at each time point, resulting in aberrant data.

Figure 5 shows a schematic diagram of a SGC in an USP Apparatus 2 dissolution vessel.

When the sampling probes were inserted into and withdrawn from the vessels, the tip filters were coated with oil. Therefore, the sample solutions which passed through these filters were not representative of drug dissolved in the aqueous phase. In order to overcome this difficulty, the sampling was done online using an Agilent UV spectrophotometer, and the sampling probes remained immersed in the dissolution medium throughout the duration of testing in order to obtain reproducible results.

Based on the solubility information (Figure 2), the drug substance appeared to have better solubility in the acetic acid/Polysorbate 80 medium than in the $0.01 \mathrm{~N} \mathrm{HCl} /$ Polysorbate 80 medium. However, the dissolution profile of Formulation A (Figure 6) showed the opposite order for the rate of dissolution.

Figure 7 shows the dissolution profiles of the two different formulations using USP Apparatus 2 in $0.01 \mathrm{~N} \mathrm{HCl}-0.25 \%$ 


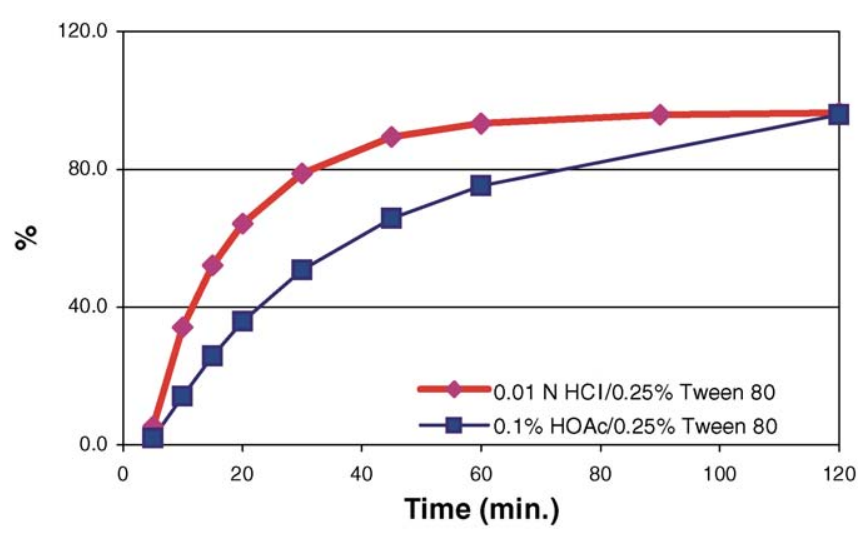

Figure 6. Dissolution Profiles of Formulation A Using USP Apparatus 2

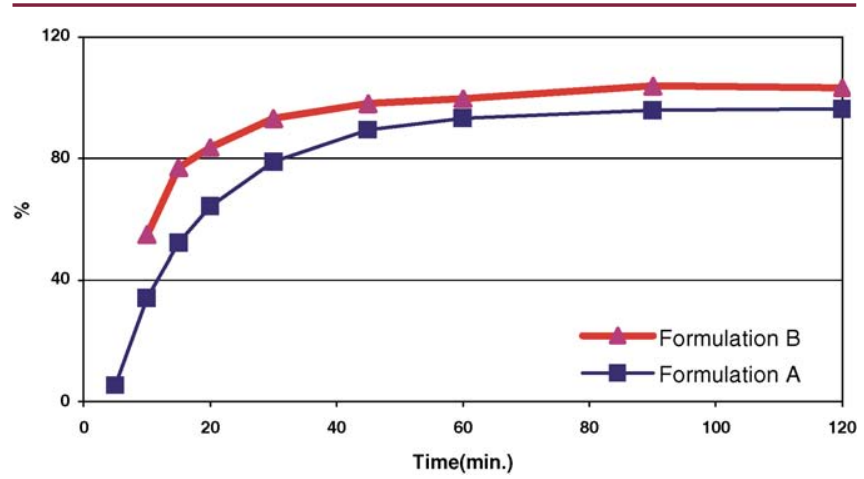

Figure 7. Dissolution Profiles of Formulation A and B using USP Apparatus 2 (Medium: 0.01N HCl/0.25\% Polysorbate 80)

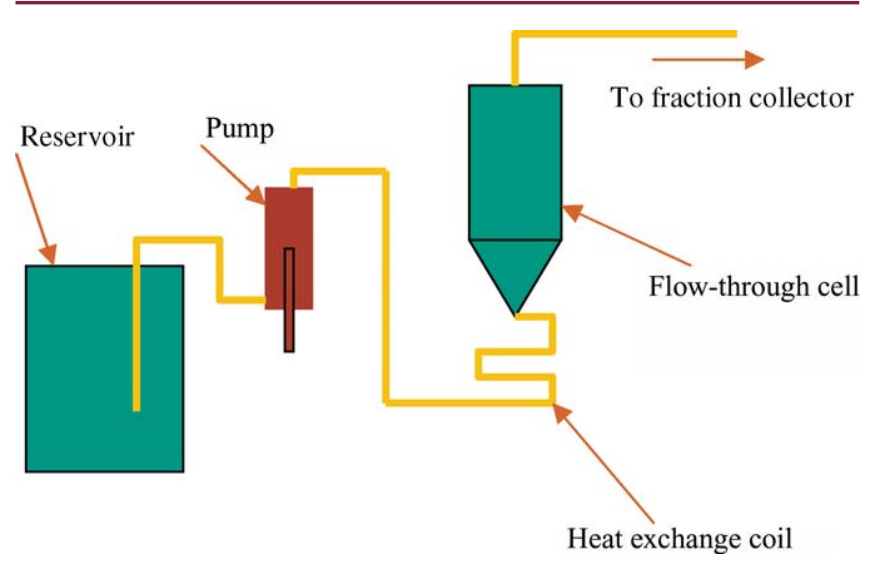

Figure 8. Flow Diagram for USP Apparatus 4 with Open System

Polysorbate 80 . The two dissolution profiles are similar and do not distinguish between the two formulations.

\section{Dissolution using USP Apparatus 4}

As an alternative to Apparatus 2, the USP Apparatus 4 (flow-through dissolution system) was considered. Apparatus 4 can be configured as an open or closed dissolution system (4). An open system was selected, and fresh dissolution medium was pumped through the flow cell continuously, thus maximizing the dissolution rate. Figure 8 shows the USP Apparatus 4 flow path for an open system.

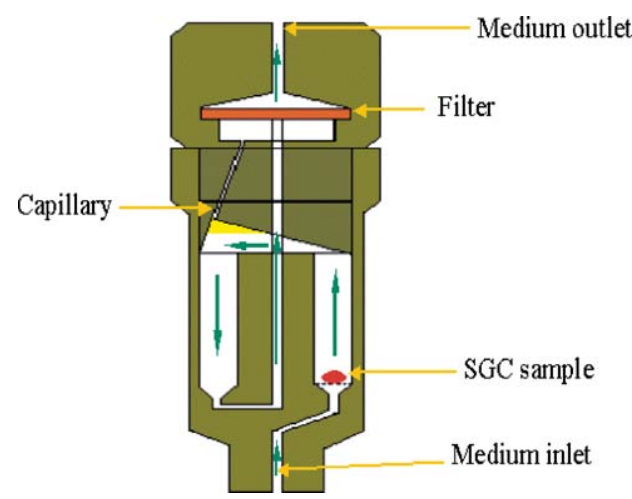

Figure 9. Detailed Diagram of the Structure of the SGC Flow-through Cell

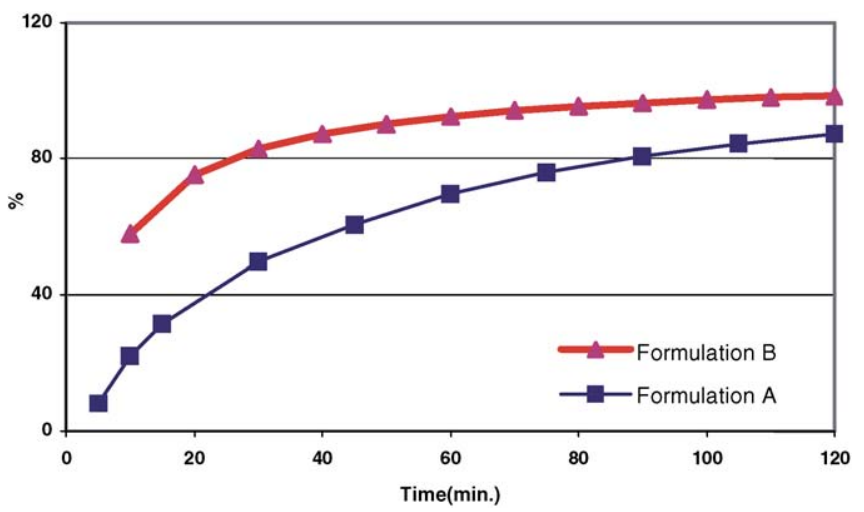

Figure 10. Dissolution Profiles of Formulations $A$ and B Using USP Apparatus 4 (Medium: $0.01 \mathrm{~N} \mathrm{HCl}-0.25 \%$ Polysorbate 80 )

The standard flow-through cell is suitable for sustainedrelease formulations and ordinary solid dosage tablet or capsule formulations. It is not suitable for lipid SGC formulations, because after rupture of the lipid SGC, the oil phase is quickly drawn into the filter on the top of the cell, which can clog the filter, or the oil is forced through the filter. Therefore, a special cell, the SGC flow-through cell (Figure 9) was selected for this analysis.

As shown in Figure 9, the SGC flow-through cell works differently from the standard flow-through cells. The dissolution medium enters through the medium inlet, on the right-hand side of the cell, going over to the left side of the cell, pushes the air out through the capillary, and then the medium flows through the center channel to the filter. After the SGC ruptures in the right-hand cell, the lipid content rises up, due to the lower density. When the lipid phase reaches the triangular area (top of the left side cell), it stays there. Thus the dissolution medium continuously extracts the drug from the lipid layer as it flows through the cell. The dissolved drug is then collected with a fraction collector and analyzed by an offline UV spectrometer.

Figure 10 shows the dissolution profiles of SGC Formulations $A$ and $B$ using USP Apparatus 4, and Figure 11 shows the dissolution profiles of Formulation $A$ obtained with four different dissolution media: 


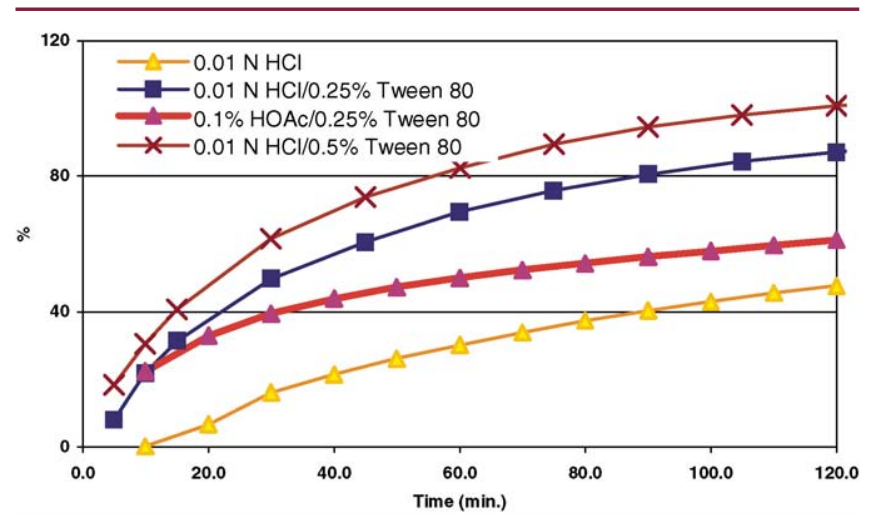

Figure 11 Dissolution Profiles of Formulation A using USP Apparatus 4 with Different Media

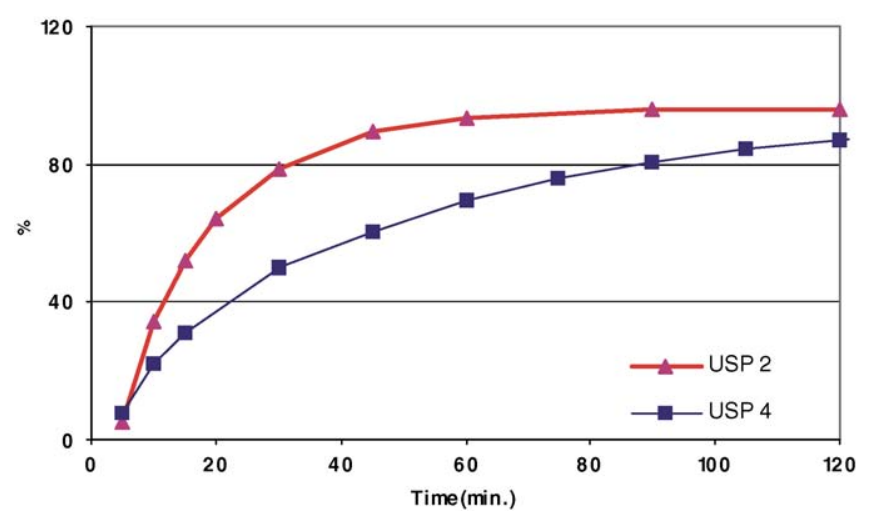

Figure 12. Comparison of Dissolution Profiles of Formulation A Using USP Apparatus 2 and USP Apparatus 4 in $0.01 \mathrm{~N} \mathrm{HCl}-0.25 \%$ Polysorbate 80

$0.01 \mathrm{~N} \mathrm{HCl} ;$

$0.01 \mathrm{~N} \mathrm{HCl} / 0.25 \%$ Polysorbate 80 ;

$0.1 \%$ acetic acid $/ 0.25 \%$ Polysorbate 80 ; and

$0.01 \mathrm{~N} \mathrm{HCl} / 0.5 \%$ Polysorbate 80

Since the $0.01 \mathrm{~N} \mathrm{HCl}$ / Polysorbate 80 medium gave the most complete drug release, it was selected for routine use.

\section{Discussion:}

The dissolution profiles obtained using USP Apparatus 2 and Apparatus 4 are compared in Figure 12. The dissolution rates obtained using USP Apparatus 2 were faster than those obtained using USP Apparatus 4 with the same dissolution medium. This faster profile was probably due to the fact that the USP Apparatus 2 gave higher agitation and mixing than Apparatus 4. The results also showed that Apparatus 4 had more discriminatory power than Apparatus 2.

Therefore, the Apparatus 4 method is more suitable to assess excipient and/or process changes that could affect the release rate of the drug from the dosage form during the formulation development.

\section{Conclusions}

The USP Apparatus 2 and Apparatus 4 were compared for dissolution of soft gelatin capsule formulations of a poorly water-soluble amine drug. Using an acidic medium with added surfactant, both apparatuses gave similar dissolution profiles. Apparatus 2 tended to give a faster rate of dissolution, but Apparatus 4 was better able to distinguish between different formulations.

When the soft gelatin capsule ruptures during dissolution, the oil phase is released into the aqueous medium. Both dissolution methods were adapted to handle the problems caused by the oil phase passing into the filters and sample collection system.

\section{References}

1. Gennadios, Aristippos. Protein-Based Films and Coatings (2002), 393-443. Publisher: CRC Press LLC, Boca Raton, Fla CODEN:69DWY6 Conference; General Review written in English.CAN 139:354205 AN 2003:346331 CAPLUS

2. Tabibi, S. Esmail;Gupta, Shanker L. Water-Insoluble Drug Formulation (2000),609-633. Publisher:Interpharm Press, Buffalo Grove, III CODEN:69AMSN Conference; General Review written in English. CAN 134:357432 AN 2000:716594 CAPLUS

3. J.Wagner, Biopharmaceutics, 17 , Rate of dissolution In Vivo and In Vitro, Part II. Drug Intell Clin Pharm. 4, 32 (1970)

4. European Pharmacopeia

Acknowledgments

Rick Chiu

Sekhar Kanapuram 